\title{
Head, Face and Neck Cooling as Per-cooling (Cooling During Exercise) Modalities to Improve Exercise Performance in the Heat: A Narrative Review and Practical Applications
}

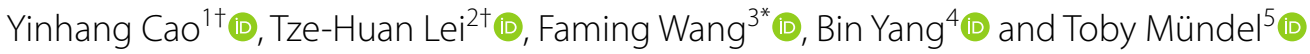

\begin{abstract}
It is well known that uncompensable heat stress greatly impairs endurance and team sport-related performance because an increase in the core temperature directly induces a greater magnitude of the central fatigue in the heat than in thermal neutral environments. Numerous studies have been conducted in an attempt to discover reliable cooling strategies for improving endurance performance and repeated sprint ability while exercising in the heat. Whole-body pre-cooling has been shown to improve endurance performance in both dry and humid heat. Despite this, the reduction in thermal perceptions associated with pre-cooling gradually narrows during intense exercise. Hence, effective per-cooling strategies to improve athletic performance in the heat are required. Unfortunately, due to practical issues, adopting pre-cooling approaches as a per-cooling (cooling during exercise) modality to improve athletic performance is impractical. Thus, we sought to examine the impact of head, neck and face cooling on athletic performance in heat. According to current evidence, cooling the head, neck and face reduced local skin temperature in the areas where cooling was applied, resulting in improved local perceptual sensations. In the heat, neck cooling during exercise improves athletic performance in both endurance and team sports athletes. Furthermore, from a practical standpoint, neck cooling is preferred over head, face and combined head/face and neck cooling for both endurance and team sport athletes in the heat. Nonetheless, for all athletes who have access to water, face cooling is a recommended cooling strategy. There is a lack of research on the systematic selection of per-cooling modalities to improve athletic performance based on environmental conditions and the nature of sports. In addition, powerful but portable head, neck and face cooling systems are urgently needed to assist athletes in improving their performance in hot conditions.
\end{abstract}

Keywords: Endurance performance, Heat stress, Thermoregulation, Pre-cooling, Per-cooling, Cooling intensity

*Correspondence: dr.famingwang@gmail.com

†Yinhang Cao and Tze-Huan Lei have contributed equally to this work

${ }^{3}$ School of Energy and Environment, Southeast University, Jiulong Lake

Campus, Nanjing 211189, China

Full list of author information is available at the end of the article

\section{Key Points}

- Cooling the neck during exercise improves endurance performance in endurance athletes in the heat. Neck cooling during exercise also improves repeated sprint performance for team sport athletes in the heat.

- Cooling the head, neck and face during exercise reduces local skin temperature and thus improves local perceptual responses. 
- Face cooling improves athletic performance and perceptual responses for athletes in dry heat. Thus, for all athletes who have access to water, face cooling is a recommended cooling strategy.

- Neck per-cooling (cooling during exercise) is preferred over head, face and combined head/face and neck per-cooling for endurance athletes.

\section{Introduction}

The current consensus $[1,2]$ clearly indicates that performing endurance exercise in uncompensable heat stress environments imposes a greater thermoregulatory strain than performing endurance exercise in a cool or thermoneutral environment. This increase in thermoregulatory strain is primarily caused by the inability to dissipate metabolic heat through both dry and evaporative heat transfer, resulting in a higher core temperature [2]. A higher core temperature increases both thermal perceptual and cardiovascular strain, resulting in voluntary power output reduction [3] or premature fatigue as measured by the time to exhaustion approach [4].

Whole-body pre-cooling with water immersion has been found to improve endurance performance in both dry and humid heat as measured by time to exhaustion $[5,6]$ or by using the self-selected pace $[7,8]$. This improvement in endurance performance is primarily due to physiological alterations (i.e., lower resting core and skin temperatures) caused by pre-cooling [5] which reduces cardiovascular and perceptual strain when performing prolonged endurance exercise in the heat. These improvements in thermoregulatory function, combined with lower perceptual strain, greatly improve endurance performance in uncompensable heat stress [9]. Nevertheless, the major drawbacks of whole-body pre-cooling are that it takes time and cannot be implemented during exercise. Furthermore, it is common that the desired physiological status (e.g., lower resting core temperature and thermal perceptions) induced by different wholebody pre-cooling regimes gradually narrows during exercise in uncompensable heat stress environments. Recently, previous studies have shown that when exercising in uncompensable heat stress (i.e., humid heat), percooling (cooling during exercise) is just as important as pre-cooling because it reduces thermal perceptions during exercise $[10,11]$. Therefore, it is now recommended that pre-cooling is used in conjunction with a per-cooling (cooling during exercise) regime when exercising in the heat.

To date, per-cooling (cooling during exercise) has been used to reduce perceptual strain during endurance or team sport events in both dry and humid heat.
Per-cooling (cooling during exercise) strategies have frequently been used to cool the upper body using a cooling vest or in local body regions such as the torso, head, face and neck. Although cooling the upper body with a cooling vest improves performance in laboratory settings, this method of per-cooling is impractical in the real-world situations due to the excessive weight of the vest (1 kg or higher). In contrast, cooling the head and neck regions is an effective way to mitigate perceptual strain and to improve physical performance in the heat and is relatively easy to wear with limited weight bearing when compared to cooling vests [12-16]. These two cooling interventions are based on the fact that the human body's head/face and neck regions have greater alliesthesial sensitivity than the rest of the body, which can result in an immediate reduction in whole-body thermal discomfort when a cold stimulus is applied to mildly heat-stressed individuals [17]. This reduction in thermal perceptions improves both endurance and repeated sprint ability in the heat without altering the thermoregulatory response [18]. Furthermore, the cooling requirements of these regions are relatively small while providing ergogenic benefits similar to those of using whole-body pre-cooling via water immersion. As a result, many practitioners around the world are grappling with how to successfully incorporate per-cooling on top of whole-body pre-cooling.

Although previous studies [10,13-15, 18] have clearly demonstrated that both head/face and neck cooling interventions can effectively improve athletic performance in the heat, those studies do not specifically address whether those interventions can be applied to both team sports and endurance athletes. Furthermore, there have been few studies that describe the physiological and performance differences between such cooling interventions. Finding a specific per-cooling strategy for different athletes is critical for recent and upcoming sporting events, e.g., the 2021 Tokyo Olympic Games and the 2022 soccer World Cup in Qatar, because it can improve physical performance while reducing thermoregulatory strain in uncompensable heat stress. The purpose of this narrative review is to specifically discuss the best per-cooling (cooling during exercise) strategies for various sports, such as endurance and team sport events. Furthermore, this narrative review provides an overview of the thermoregulatory and perceptual responses of each cooling intervention, which may provide valuable recommendations to the public regarding which cooling intervention is preferred when performing endurance exercise or team sports events during the summer. Finally, we highlight unexplored issues that could spur more mechanistic research in this field. 


\section{Literature Search Methods and Considerations}

Literature searches were conducted in the PubMed, MEDLINE, Scopus, Google Scholar and ProQuest databases until September 2021. Keywords used in the search included neck cooling, carotid cooling, head cooling, face cooling, exercise, per-cooling, pre-cooling, post-cooling, personal cooling, endurance performance, repeated sprint and time trial. Studies were included if the following criteria were met:

- Participants were described as "healthy" or "active," and there were no known diseases that could impair exercise performance or thermoregulation;

- Studies were conducted at a temperature of $\geq 20^{\circ} \mathrm{C}$;

- Studies examined at least one type of cooling strategy applied to the head, face or neck or any possible combination of these three body regions;

- Studies reported either physiological or perceptual responses, or both;

- Studies were published in English in peer-reviewed journals, conference proceedings or published theses.

We excluded studies that used head, face and/or neck cooling modalities during occupational activities such as firefighting, farming or construction work. Any study that did not report the test condition was also disqualified. We also excluded two older studies from the 1970s and 1980s because we did not have access to the full texts and were unable to contact the authors to obtain the full texts.

Our search yielded 32 results for "neck cooling" AND "exercise" AND "healthy", 24 results for "face cooling" AND "exercise" AND "healthy," 22 results for "head cooling" AND "exercise" AND "healthy," 15 results for "head and face cooling" AND "exercise" AND "healthy," 9 results for "head and neck cooling" AND "exercise" AND "healthy" and 7 results for "face and neck cooling" AND "exercise." Sixty-four of the 109 references did not address the areas of interest. Five relevant conference proceeding articles/extended abstracts and 1 relevant published thesis were discovered in the Google Scholar and ProQuest databases. Ultimately, 51 studies were included in this narrative review.

Figure 1 depicts the co-occurrence map of the most frequently used keywords in the abstracts of the 51 studies using VOSviewer (software version 1.6.17, Leiden University, The Netherlands). The co-occurrence map depicts the extent to which keywords occur concurrently in the reviewed keyword volume. The connection between any two concurrent keywords is represented by the network, while the frequency of a specific keyword is represented by its circle size. The dense network shows that keywords such as "male," "female," "face," "head," "neck," "body temperature," "skin temperature" and "heart rate" were frequently used and correlated with almost every other keyword. Furthermore, "adult" subjects were used as research subjects in the vast majority of documented studies. Furthermore, physiological parameters have been extensively studied, but there is very little information on perceptual responses such as thermal sensation (TS), thermal comfort/discomfort, thermal acceptance and ratings of perceived exertion. Future percooling studies should include females and young adults or even aged athletes as research participants. In addition to physiological parameters, perceptual responses while using per-cooling devices should be systematically examined and reported.

\section{Neck Cooling and Exercise Performance}

Table 1 summarizes the published research on neck cooling and its performance on athletes while exercising in heat $[13-15,18-36]$. Cooling collars, wet towels and ice bags were the most commonly used neck cooling approaches in the 23 documented studies. First, although six out of 23 studies did not report local neck temperature, the use of neck cooling could greatly reduce local skin temperature at the neck, and thus local thermal sensation at the neck was also significantly improved. A neck cooling collar with a $-80{ }^{\circ} \mathrm{C}$ cooling refrigerant has been found to improve both endurance and team sport performance in heat $[13-15,18,19,28-30]$. This is demonstrated by the fact that wearing a cold neck collar improves time to exhaustion, self-paced performance and repeated sprint ability $[13-15,18,19]$ when compared to no-cooling trials. This increase in physical performance with neck cooling may be due to the neck region's high alliesthesial sensitivity and close proximity to the thermoregulatory center, namely, the insular cortex, which means that any cold stimulus can directly result in an immediate change in local thermal sensation (TS) and thus the rate of perceived exertion [17]. As a result, the self-selected pace or time to reach volitional exhaustion increases. The improved endurance performance with a neck cooling collar is primarily perceptually mediated, and its $T_{\text {core }}$ response differs between time to exhaustion and the self-selected pace approach.

When assessing endurance performance using the time-to-exhaustion approach, the time to volitional exhaustion with the neck cooling collar was significantly longer than that in the control trial. This, however, corresponds to a higher core temperature at the time of exhaustion [14], leading to the conclusion that neck cooling may increase the risk of heat-related illness by masking the extent of thermal strain perceived. However, this statement is debatable because this approach does not allow us to thermoregulate behavior, which is 


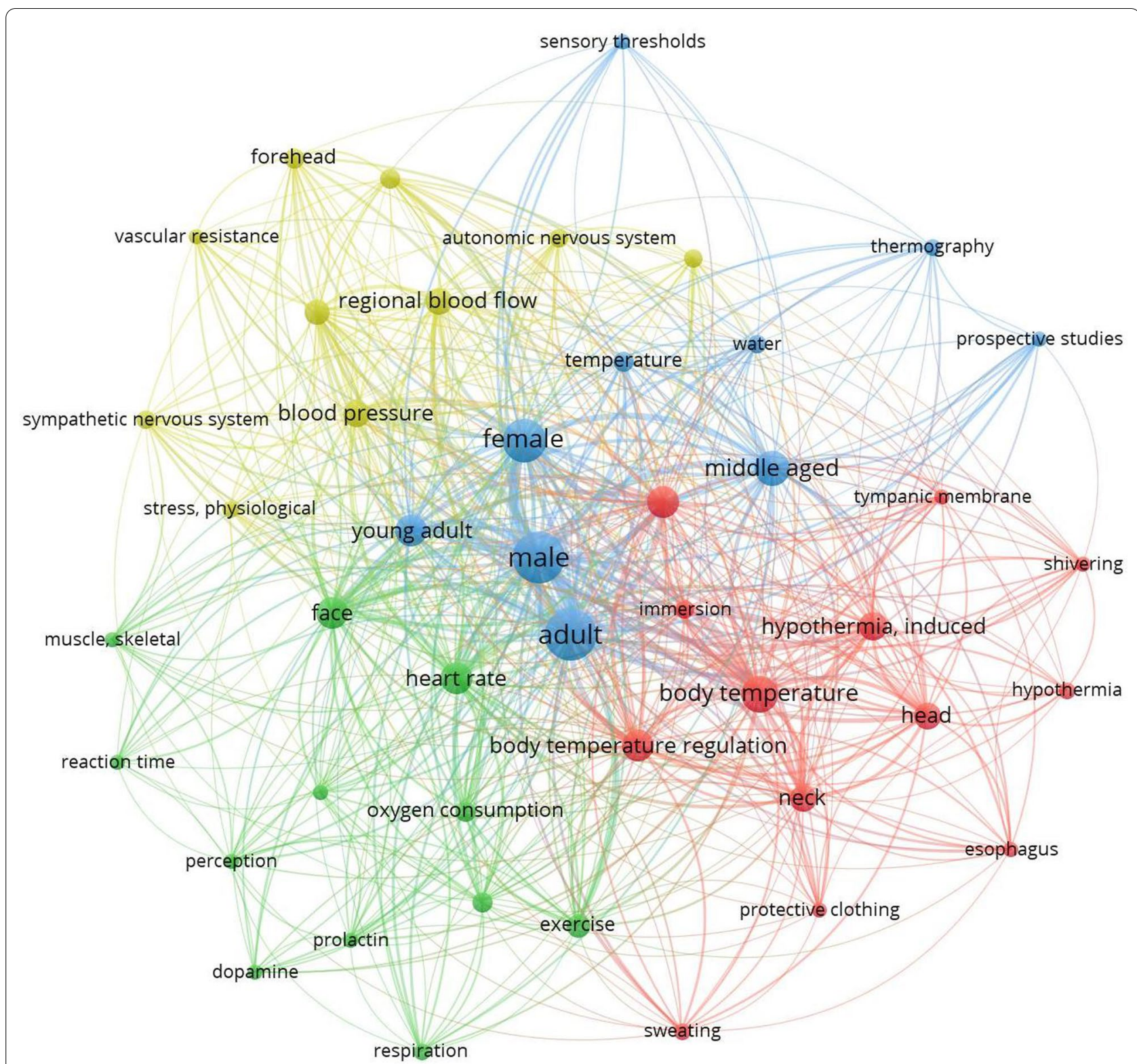

Fig. 1 Co-occurrence map of frequently used keywords from the 51 published references

an important mechanism for controlling body temperature during exercise. Furthermore, time to exhaustion has poor face validity and cannot represent the actual endurance event due to its open-ended design. Neck cooling has been shown in studies using self-selected pace to increase overall power output without affecting core temperature, heart rate and neuroendocrinological response $[13,15]$. This suggests that by using our behavioral response, we can counteract the false signal caused by neck cooling and thus avoid an excessive rise in core temperature in the heat. Therefore, neck cooling is an effective cooling method for improving the endurance performance in the heat. However, it is worth noting that neck cooling may not be effective if the duration of exercise is less than $15 \mathrm{~min}$, as Tyler et al. [13] demonstrated that neck cooling was ineffective in improving physical performance in a 15 -min time trial.

It is also worth mentioning that some studies (13 out of 23, Table 1) [13, 20-25, 31-36] show that neck cooling does not provide any ergogenic benefit when performing prolonged exercise in the heat. The large disparity can be attributed to the cooling intensity not being strong enough to change the rating of perceived exertion (RPE), which is widely thought to be the most important 


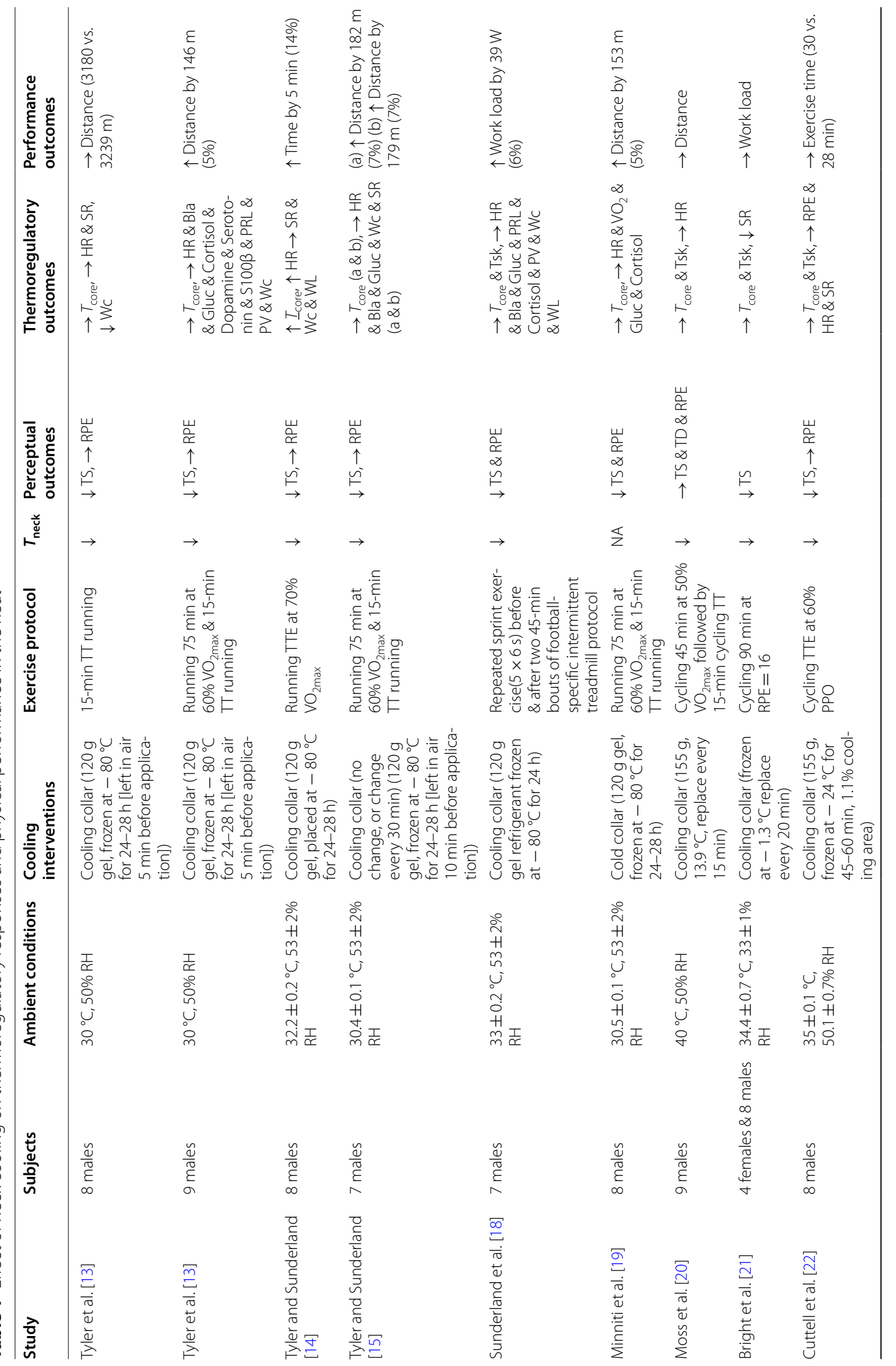




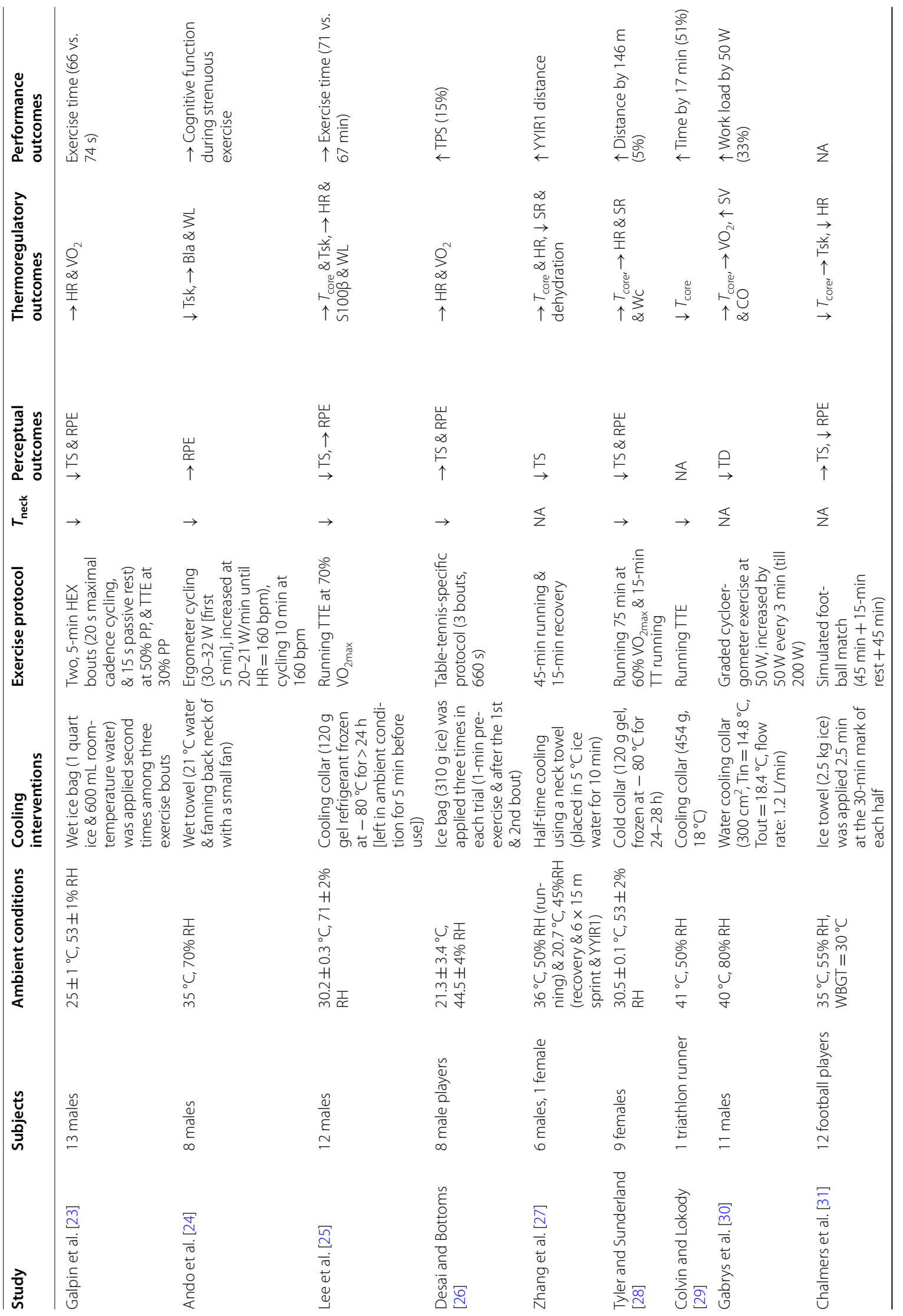




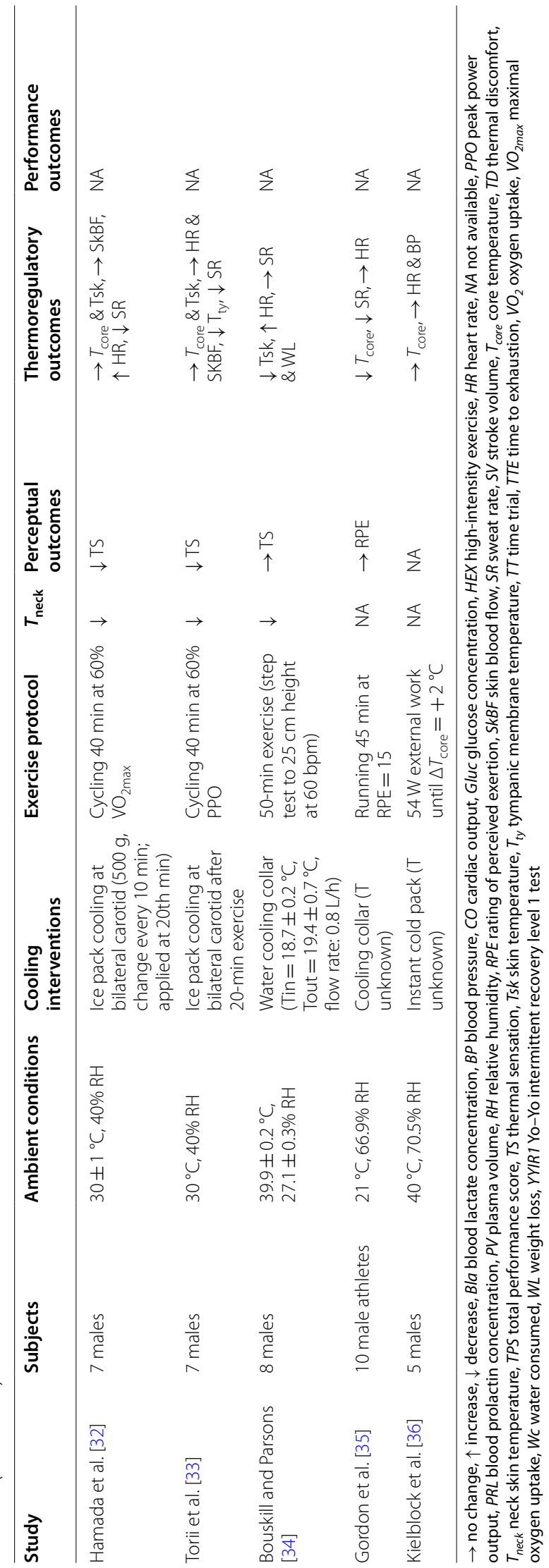


perceptual marker for determining central fatigue in the heat because it is directly correlated with arousal level [68]. Specifically, the $\alpha / \beta$ wave ratio increased during hyperthermia, which has been linked to decrease arousal levels and contributes to the inability to maintain the desired power output during prolonged exercise in the heat.

\section{Head Cooling and Exercise Performance}

A summary of 11 published studies on head cooling and its impact on sports and exercise performance is listed in Table 2 [16, 37-45]. Surprisingly, only four studies examined local head temperature and all discovered a pronounced temperature reduction at the head. More than half of the studies (6 out of 11 studies) did not investigate athletes' perceptual responses during head cooling. Published studies [38-41] suggested that head cooling could potentially improve endurance performance in the heat [38-41], but this may not apply to team sport athletes because current studies (see Table 2) have yet to investigate whether head cooling can potentially improve repeated sprint ability for team sport athletes in any given thermal environment. Furthermore, whether head cooling can improve endurance performance in heat is still debatable, with the majority of studies revealing that head cooling has no ergogenic benefits in heat. There are currently very few studies on the effect of head cooling on the self-selected pace in the heat. Such disparities in previous studies are related to the intensity of the cooling, which is directly related to the cooling materials being used inside the cooling cap or the cooling helmet. It is possible that the majority of previous studies used a low cooling intensity for their study design, which was insufficient to alter the RPE response during exercise in the heat. Conversely, some studies (Table 2) have found that when the cooling intensity $\left(5^{\circ} \mathrm{C}\right.$ of water) is able to attenuate the rise of RPE, physical performance improves $[16,40]$. Therefore, it is believed that head cooling with a higher cooling intensity could potentially alter our arousal level, affecting our physical performance. This particular notion, however, necessitates further justification.

\section{Face Cooling and Exercise Performance}

Table 3 shows the results of face cooling and its performance. Nine of the eleven included studies examined either the forehead or the face temperature. Six studies $[12,46,49-51,54]$ examined local perceptual sensations (thermal sensation and thermal discomfort). Face cooling, such as the effect of neck and head cooling on the neck and head regions, has the potential to greatly improve thermal sensation and alleviate thermal discomfort at the face. For physiological outcomes, although three studies [12, 46, 55] have suggested that face cooling appears to improve athletic performance in the heat (Table 3), the effect of face cooling on prolonged endurance performance needs further investigation. This is supported by the fact that face cooling only reduced RPE at the end of the stage [56]. Such small decrements of RPE may not be sufficiently large to elicit behavioral adaptation in highly trained subjects as their perceptual responses differ from those of the untrained population [57]. Stevens et al. [46] found that even in moderately trained subjects, face cooling improved the self-paced running speed for the first $2 \mathrm{~km}$ but was soon nullified thereafter. Nonetheless, intermittent face cooling significantly improved forehead temperatures, thermal sensation and muscle activation during the $5-\mathrm{km}$ running time trial. Furthermore, no research has been conducted to determine whether face cooling can potentially increase the time to exhaustion or time trial performance in welltrained endurance athletes. Team sport athletes appear to reap significant ergogenic benefits from face cooling, as Miyazawa et al. [54] demonstrated that intermittent face cooling, similar to the team sport situation, is capable of lowering RPE during exercise in heat. Riera et al. [55] provided additional evidence that face cooling during swimming could increase the peak swimming velocity during 400-m swim events. However, it is worth noting that cooling the face with room temperature water $\left(22{ }^{\circ} \mathrm{C}\right)$ had little effect on athletic performance, whereas cooling the face with cold water $\left(1.2{ }^{\circ} \mathrm{C}\right)$ greatly improved the peak swimming velocity [55]. However, it remains unknown whether face cooling with water can provide ergogenic benefits to athletes in humid heat [58]. It is possible to conclude that face cooling improves athletic performance and perceptual responses for athletes in dry heat. Thus, for all athletes who have access to water, face cooling is a recommended cooling strategy. Nonetheless, to maximize face cooling benefits, the water temperature and the frequency of intermittent face cooling should be carefully chosen.

\section{Combined Head, Neck and/or Face Cooling and Exercise Performance}

Table 4 shows the effects of combined face/head and neck cooling on sports and exercise performance [56, 59-64]. Clearly, combined head/face and neck cooling could largely improve thermal sensation and thermal discomfort at the cooling regions [56, 59-64]. The combined effect of either head and neck cooling or face and head cooling on exercise performance in the heat is unclear. Despite featuring a larger total cooling area in the combined cooling strategy when compared to the single cooling approach being applied to the face, neck or head, these aforementioned combined cooling methods 


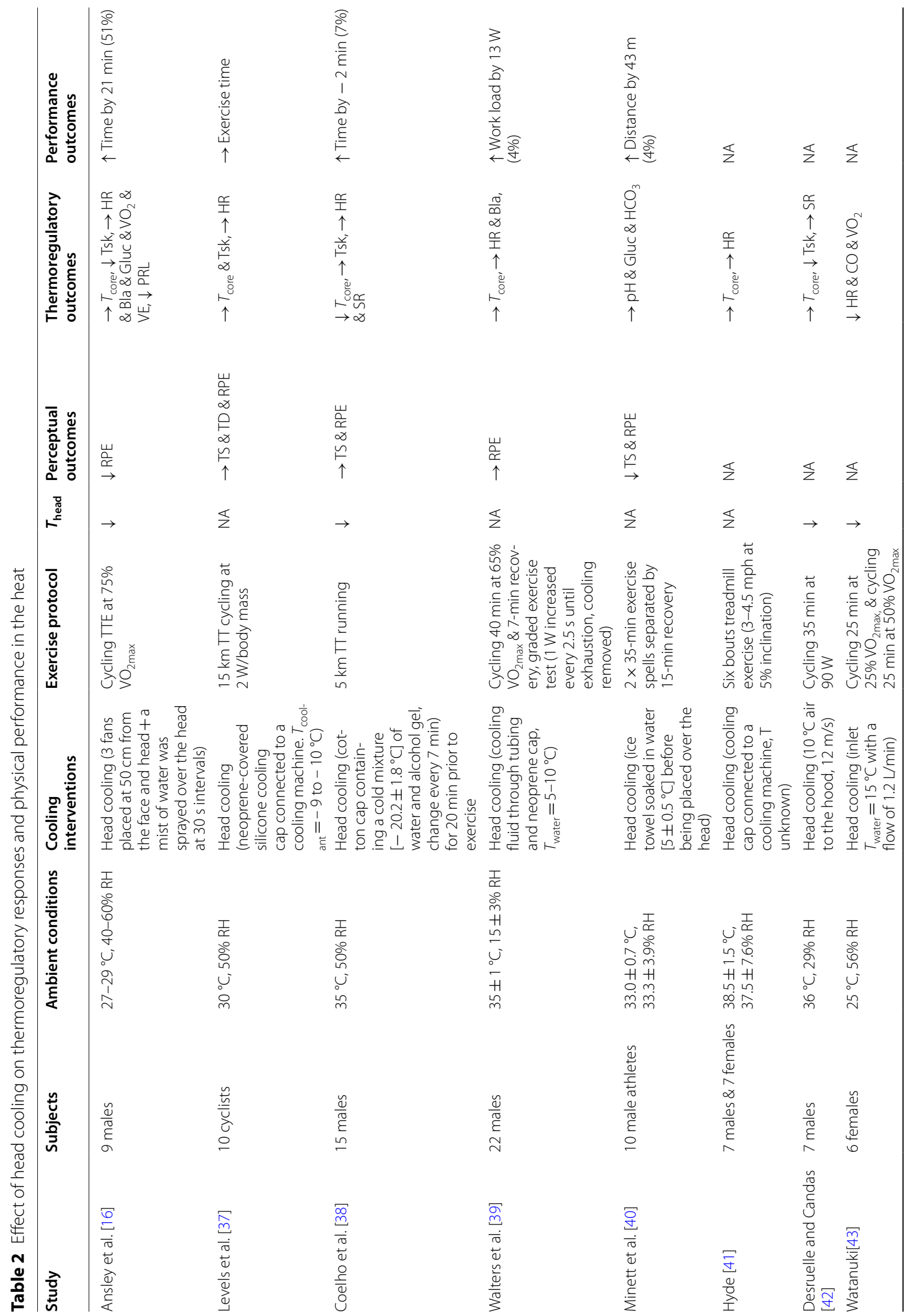




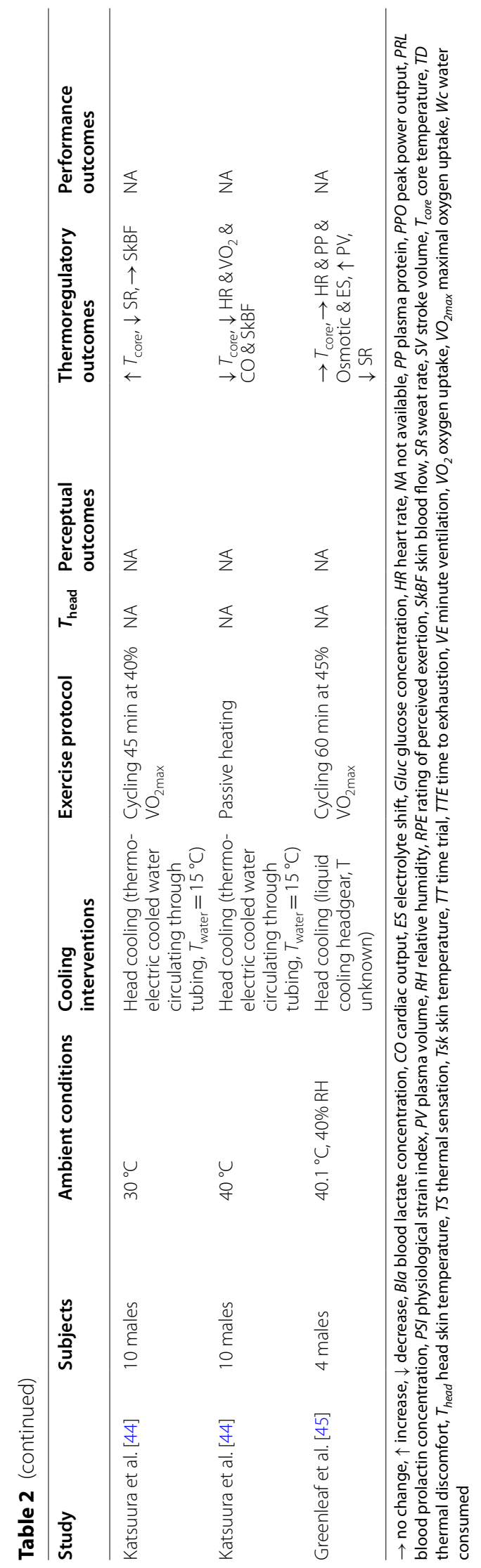




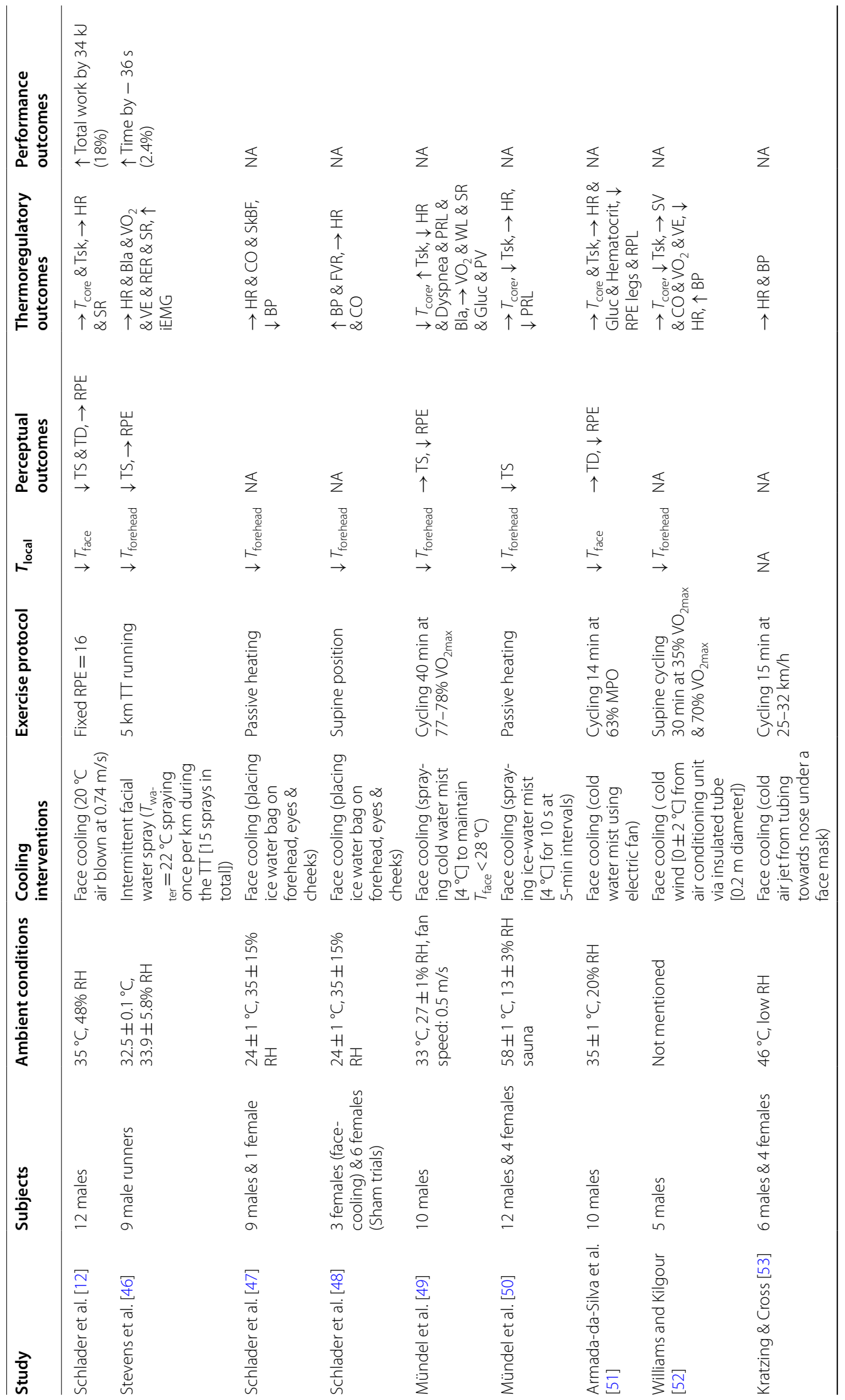




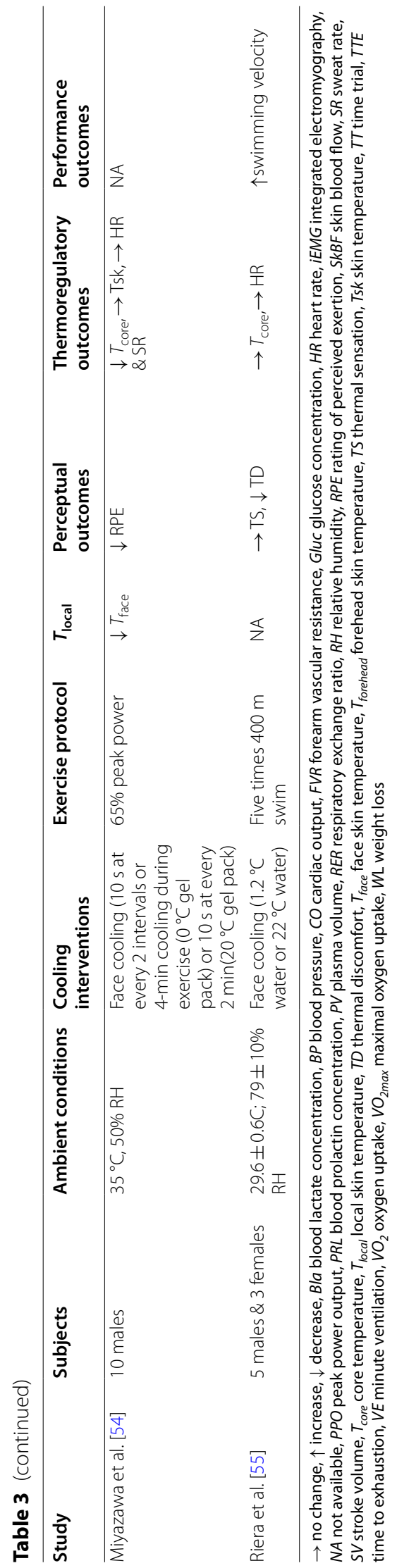




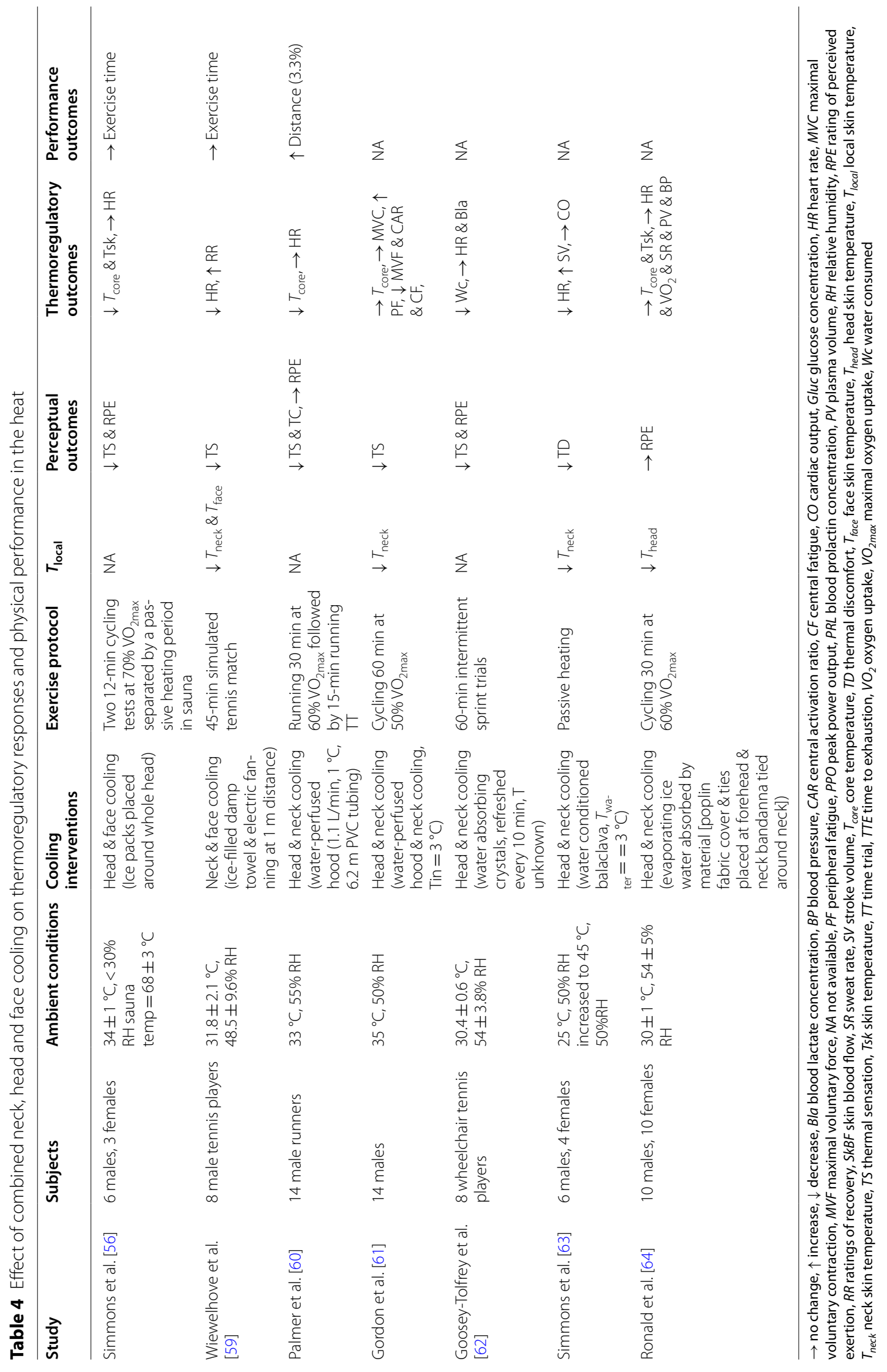


may not provide any ergogenic benefits when compared to neck cooling alone [65]. The current literature has yet to address the combined effect of either head and neck cooling or face and head cooling on exercise performance in heat when compared to neck cooling alone for both endurance and team sport athletes. Furthermore, summarizing the current literature (Tables 1, 2, 3), it is suggested that the combined cooling may not actually provide any ergogenic benefit because the effect of both head and face cooling on both endurance and repeated sprint performance is equivocal. Finally, from a practical standpoint, combined cooling is thought to have less real-world application than neck cooling alone due to the difficulty of implementing this cooling intervention during exercise in the heat.

Although the combined cooling intervention may not be appropriate as a per-cooling strategy for both endurance and team sport athletes in the heat, it may be effective as a post-cooling strategy after performing exercise in the heat because it may compensate for the withdrawal of the thermoeffector, thereby reducing the magnitude of post-exercise hyperthermia. In particular, this intensive cooling intervention may benefit occupational workers wearing protective clothing or American football players wearing multiple layers of body armor more than neck cooling alone. However, this separate and combined effect has not been investigated and thus warrants further investigation.

\section{Practical Considerations}

Summarizing the current literature to date (Tables 1, 2, $3,4)$, neck cooling is preferred over head or face cooling because it can be used before and during exercise in the heat for both endurance and team sport athletes [66]. In addition, head cooling is preferred over facial cooling because it can also be used as a pre- and per-cooling strategy in the heat. It is worth mentioning that the cooling temperature selected from the neck cooling collar or cooling helmet must be individualized because each individual will have their own preferred cooling temperature, but the cooling temperature must be sufficient to alter the RPE without causing a further increase in core temperature. Furthermore, it is recommended that endurance athletes use either a neck or head cooling intervention following the completion of their race to compensate for the effect of post-exercise hyperthermia, especially when the heat stress environment is hot and humid [67].

Last, it is suggested that the cooling materials inside the cooling collar or the helmet should include phase change materials (including ice) rather than gel refrigerants because phase change materials provide a more stable cooling intensity during phase change than gel refrigerants [68]. Although soft gel could provide good flexibility when applied to the body surface, its temperature rises throughout the entire application period. Soft gel refrigerants, in particular, provide the most powerful cooling intensity at first, but this cooling power gradually diminishes over time. Furthermore, the mass of phase change materials should be large to provide athletes with prolonged cooling during exercise. Other cooling strategies such as the use of wearable cooling fans and a liquid cooling neck collar may be applicable; however, their actual effectiveness on performance improvement in exercising athletes requires further investigation.

\section{Directions for Future Research}

While current studies have eloquently addressed the effects of neck, head, face as well as the combined head and neck cooling on both endurance and team sport performance, there are three major unexplored issues that warrant further investigation. First, recent studies have not specifically included evaluations of the effects of different cooling modes on local and whole-body thermal discomfort, thermal pleasantness and skin wettedness in their research design. It is well known that thermal perceptions can influence RPE, thereby affecting our physical performance in the heat $[12,69]$. Furthermore, because those perceptual variables are important in determining our thermoregulatory behavior [12, 70, 71], it would be novel to investigate whether cooling per se would mask the signal to initiate our thermoregulatory behavior, which has an unlimited capacity to regulate our body temperature.

Second, previous studies have not taken into account the role of aerobic fitness in the effect of neck, head, face and combined head and neck cooling during self-paced exercise in heat. When exercising in the heat, welltrained populations are more accustomed to thermal discomfort [72] than untrained individuals, and thus, additional cooling may not be ergogenic when compared to untrained individuals. Indeed, previous studies primarily targeted endurance or team sport athletes, which could explain why some studies found no performance benefit from using neck cooling during exercise.

Next, the rationale for selecting cooling devices in a specific sports and exercise setting has not yet been thoroughly investigated. For instance, the temperature of the cooling device varies considerably, ranging from 21 to $-80{ }^{\circ} \mathrm{C}$, and such variations in cooling temperature would undoubtedly alter our thermal perceptions and thus our exercise performance in the heat. Furthermore, to date, the selection of cooling temperatures is not evidence-based; rather, they are chosen without any supporting evidence for either thermoregulatory or perceptual response to heat. Additionally, the mass of cooling materials, the type of cooling strategy (e.g., air cooling, 
phase change material cooling, evaporative cooling, and liquid cooling) and the design of the cooling devices (e.g., packing material, insulation between the cooling material and human skin, and contact area) are unclear. It is also well known that exposure to high levels of solar radiation reduces endurance capacity and raises skin temperature during heat-related exercise. Nonetheless, previous cooling studies did not take into account the effects of sunlight on cooling during exercise-heat stress. Furthermore, under such conditions, the cooling materials (phase change materials and gel refrigerant) melt faster than in the absence of sun exposure. The aforementioned factors may have a significant impact on the cooling intensity and the cooling duration of the chosen device. As a result, their actual impact on performance enhancement while exercising in different heat stress environments may vary greatly. Therefore, the aforementioned issues merit further investigation because they would broaden our understanding of the effects of per-cooling in both compensable and uncompensable heat stress environments. Furthermore, only 12 out of 49 studies (see Tables 1, 2, $3,4)$ included females as exercising participants. Females have very different thermal and perceptual responses to exercise heat stress across their menstrual cycle [73]. As a result, more research is needed in this area to investigate sex differences in thermal responses to head, face and neck cooling.

Last, in addition to the head, neck and face cooling discussed in this narrative review, other per-cooling (cooling during exercise) strategies, such as palm cooling, have also been used to mitigate heat strain in athletes during heat. Human hands, in general, have the second highest cold thermosensitivity after the human facial regions (including the neck) [74]. The proximal regions of the hands, such as the palm and sole, are much more thermally sensitive than the rest of the hand regions [75]. Currently, only four studies on the use of palm cooling to improve exercise performance in heat have been found. According to Grahn et al., cooling the palm increased the endurance exercise duration by $42.7 \%$ and attenuated the temperature rise in the esophagus [76]. The effect of cooling on exercise endurance decreased as the workload increased. Grahn et al. [77] discovered that palm cooling could improve high-intensity resistive exercise, but the cooling benefits are dependent on many factors, including fitness levels, inherent athletic ability, and the types and durations of the training regimes. Palm cooling is recommended as a useful strategy for athletes to maximize the training effects of resistive exercise. Despite the abovementioned ergogenic benefits of palm cooling for subjects, two studies [78, 79] found conflicting results after cooling subjects at the palm in the heat. Amorim et al. [78] and Scheadler et al. [79] observed that palm cooling does not reduce thermal strain or improve endurance performance during exercise. Several marathon runners and race walkers ran with ice packs in their hands during the Tokyo 2020 Olympic Games and the Doha 2019 IAAF (International Association of Athletics Federations) World Athletics Championships. It is unclear whether palm cooling, such as by hand-holding ice packs, can improve endurance performance because existing studies did not use the time trial approach or the time to exhaustion in well-trained athletes. This possibility merits further investigation. In addition, no research has been conducted to investigate the effect of palm cooling on athletes' perceptual responses. More research is needed to investigate this effect.

\section{Conclusions}

Neck, face and head cooling could significantly reduce local skin temperature, improving local perceptual sensations such as thermal sensation and thermal discomfort in athletes exercising in the heat. For both endurance and team sport athletes, neck cooling is a better per-cooling strategy than head or face cooling. Furthermore, while head cooling is preferred over face cooling for endurance athletes, its effectiveness among team sport athletes has yet to be determined. For all athletes who have access to water, face cooling is a recommended cooling strategy. Furthermore, it is unclear whether the combined cooling of the head, neck and/or face could have a synergistic effect on sports and exercise performance in the heat. Future research should thoroughly investigate the potential of neck, head and face cooling to improve the performance of male and female athletes, while they participate in various types of sports and exercise activities in heat. Furthermore, the development of powerful but portable head, neck and face cooling systems is critical due to the great need to dissipate metabolic heat production during intense sports activities.

\footnotetext{
Abbreviations

Bla: Blood lactate concentration; BP: Blood pressure; CAR: Central activation ratio; CF: Central fatigue; CO: Cardiac output; FVR: Forearm vascular resistance; Gluc: Glucose concentration; HEX: High-intensity exercise; HR: Heart rate; IAAF: International Association of Athletics Federations; iEMG: Integrated electromyography; MVC: Maximal voluntary contraction; MVF: Maximal voluntary force; NA: Not available; PF: Peripheral fatigue; PPO: Peak power output; PRL: Blood prolactin concentration; PSI: Physiological strain index; PV: Plasma volume; RER: Respiratory exchange ratio; RH: Relative humidity; RPE: Rating of perceived exertion; RR: Ratings of recovery; SkBF: Skin blood flow; SR: Sweat rate; SV: Stroke volume; $T_{\text {core }}$ : Core temperature; TD: Thermal discomfort; $T_{\text {face: }}$ : Face skin temperature; $T_{\text {forehead: }}$ Forehead skin temperature; $T_{\text {head }}$ : Head skin temperature; $T_{\text {local }}$ : Local skin temperature; $T_{\text {neck: }}$ Neck skin temperature; TPS: Total performance score; TS: Thermal sensation; Tsk: Skin temperature; $T_{\text {ty: }}$ : Tympanic membrane temperature; TT:Time trial; TTE: Time to exhaustion; VE: Minute ventilation; $\mathrm{VO}_{2}$ : Oxygen uptake; $\mathrm{VO}_{2 \text { max }}$ : Maximal oxygen uptake; Wc: Water consumed; WL: Weight loss; YYIR1:Yo-Yo intermittent recovery level 1 test.
} 


\section{Authors' Contributions}

F.W. provided the original idea; F.W., T.H.L, Y.C. and B.Y. helped in development and formulation of concept; Y.C., T.H.L., F.W. drafted the manuscript; critical revision was performed by T.H.L., F.W., B.Y. and T.M. All authors read and approved the final version of the manuscript.

\section{Funding}

This work was supported by Shanghai Key Lab of Human Performance at Shanghai University of Sport (Grant No.: 11DZ2261100).

\section{Availability of Data and Materials}

Data are available on request from the authors.

\section{Code Availability}

Not applicable.

\section{Declarations}

\section{Ethics Approval and Consent to Participate}

Not applicable.

\section{Consent for Publication}

Not applicable.

\section{Competing interest}

Yinhang Cao, Tze-Huan Lei, Faming Wang, Bin Yang and Toby Mündel declare that they have no conflicts of interest relevant to the content of this review.

\begin{abstract}
Author details
${ }^{1}$ School of Physical Education and Sport Training, Shanghai University of Sport, Shanghai 200438, China. ${ }^{2}$ College of Physical Education, Hubei Normal University, Huangshi 435002, China. ${ }^{3}$ School of Energy and Environment, Southeast University, Jiulong Lake Campus, Nanjing 211189, China. ${ }^{4}$ School of Energy and Safety Engineering, Tianjin Chengjian University, Tianjin 300384, China. ${ }^{5}$ School of Sport, Exercise and Nutrition, Massey University, Palmerston North, New Zealand.
\end{abstract}

\section{Received: 12 August 2021 Accepted: 16 January 2022}

Published online: 29 January 2022

\section{References}

1. Racinais S, Alonso J-M, Coutts AJ, Flouris AD, Girard O, González-Alonso $J$, et al. Consensus recommendations on training and competing in the heat. Scand J Med Sci Sports. 2015;25:6-19.

2. Sawka MN, Leon LR, Montain SJ, Sonna LA. Integrated physiological mechanisms of exercise performance, adaptation, and maladaptation to heat stress. Compr Physiol. 2011;1(4):1883-928.

3. Cheuvront SN, Kenefick RW, Montain SJ, Sawka MN. Mechanisms of aerobic performance impairment with heat stress and dehydration. J Appl Physiol. 1985;109(6):1989-95.

4. Flouris AD, Schlader ZJ. Human behavioral thermoregulation during exercise in the heat. Scand J Med Sci Sports. 2015;25(Suppl 1):52-64.

5. González-Alonso J, Teller C, Andersen SL, Jensen FB, Hyldig T, Nielsen B. Influence of body temperature on the development of fatigue during prolonged exercise in the heat. J Appl Physiol. 1999;86(3):1032-9.

6. Hasegawa H, Takatori T, Komura T, Yamasaki M. Combined effects of precooling and water ingestion on thermoregulation and physical capacity during exercise in a hot environment. J Sports Sci. 2006;24(1):3-9.

7. Booth J, Marino F, Ward JJ. Improved running performance in hot humid conditions following whole body precooling. Med Sci Sports Exerc. 1997;29(7):943-9.

8. Kay D, Taaffe DR, Marino FE. Whole-body pre-cooling and heat storage during self-paced cycling performance in warm humid conditions. J Sports Sci. 1999;17(12):937-44.

9. Marino F. Methods, advantages, and limitations of body cooling for exercise performance. Br J Sports Med. 2002;36(2):89-94.
10. Stevens CJ, Taylor L, Dascombe BJ. Cooling during exercise: an overlooked strategy for enhancing endurance performance in the heat. Sports Med. 2017;47(5):829-41.

11. Hasegawa H, Takatori T, Komura T, Yamasaki M. Wearing a cooling jacket during exercise reduces thermal strain and improves endurance exercise performance in a warm environment. J Strength Cond Res. 2005;19(1):122-8

12. Schlader ZJ, Simmons SE, Stannard SR, Mündel T. The independent roles of temperature and thermal perception in the control of human thermoregulatory behavior. Physiol Behav. 2011;103(2):217-24.

13. Tyler CJ, Wild P, Sunderland C. Practical neck cooling and time-trial running performance in a hot environment. Eur J Appl Physiol. 2010;110(5):1063-74

14. Tyler CJ, Sunderland C. Cooling the neck region during exercise in the heat. J Athl Train. 2011;46(1):61-8.

15. Tyler CJ, Sunderland C. Neck cooling and running performance in the heat: single versus repeated application. Med Sci Sports Exerc. 2011:43(12):2388-95.

16. Ansley L, Marvin G, Sharma A, Kendall MJ, Jones DA, Bridge MW. The effects of head cooling on endurance and neuroendocrine responses to exercise in warm conditions. Physiol Res. 2008;57(6):863-72.

17. Cotter JD, Taylor NA. The distribution of cutaneous sudomotor and alliesthesial thermosensitivity in mildly heat-stressed humans: an open-loop approach. J Physiol. 2005;565:335-45.

18. Sunderland C, Stevens R, Everson B, Tyler CJ. Neck-cooling improves repeated sprint performance in the heat. Front Physiol. 2015;6:314.

19. Minniti A, Tyler CJ, Sunderland C. Effects of a cooling collar on affect, ratings of perceived exertion, and running performance in the heat. Eur J Sport Sci. 2011;11(6):419-29.

20. Moss JN, Trangmar SJ, Mackenzie RWA, Tyler CJ. The effects of pre- and per-cooling interventions used in isolation and combination on subsequent 15-min time-trial cycling performance in the heat. J Sci Med Sport. 2021;24(8):800-5.

21. Bright FM, Chaseling GK, Jay O, Morris NB. Self-paced exercise performance in the heat with neck cooling menthol application, and abdominal cooling. J Sci Med Sport. 2019;22:371-7.

22. Cuttell SA, Kiri V, Tyler C. A comparison of 2 practical cooling methods on cycling capacity in the heat. J Athl Train. 2016;51:525-32.

23. Galpin AJ, Bagley JR, Whitcomb B, Wiersma LD, Rosengarten J, Coburn JW, Judelson DA. Effects of intermittent neck cooling during repeated bouts of high-intensity exercise. Sports. 2016;4:38.

24. Ando S, Komitama T, Sudo M, Kiyonaga A, Tanaka H, Higaki Y. The effects of temporal neck cooling on cognitive function during strenuous exercise in a hot environment: a pilot study. BMC Res Notes. 2015;8:202.

25. Lee JKW, Koh ACH, Koh SXT, Liu GJX, Nio AQX, Fan PWP. Neck cooling and cognitive performance following exercise-induced hyperthermia. Eur J Appl Physiol. 2014;114:375-84.

26. Desai T, Bottoms L. Neck cooling improves table tennis performance amongst young national level players. Sports. 2017;5:19.

27. Zhang Y, Nepocatych S, Katica CP, et al. Effect of half time cooling on thermoregulatory responses and soccer-specific performance test. Monten J Sports Sci Med. 2014;1:17-22.

28. Tyler CJ, Sunderland C. Neck cooling during exercise in the heat improves subsequent treadmill time-trial performance. Med Sci Sport Exerc. 2008:40:S368

29. Colvin DP, Lokody T. Development of a macroPCM neck cooling collar for athletes and runners. In: Proceedings of IMECE'03, ASME international mechanical engineering congress, Washington DC, No. 15-21; 2003. pp. $1-2$.

30. Gabryś J, Pieniazek W, Olejnik I, Pogorzelska T, Karpe J. Effects of local cooling of the neck on circulatory responses in men subjected to physical exercise in hyperthermia. Biol Sport. 1993;10:167-71.

31. Chalmers S, Siegler J, Lovell R, Lynch G, Gregson W, Marshall P, Jay O. Brief in-play cooling breaks reduce thermal strain during football in hot conditions. J Sci Med Sport. 2019;22:912-7.

32. Hamada S, Torii M, Szygula Z, Adachi K. Effect of partial body cooling on thermophysiological responses during cycling work in a hot environment. J Therm Biol. 2006:31:194-207.

33. Torii M, Adachi K, Miyabayashi T, Arima T, Iwashita M. Effect of bilateral carotid cooling with an ice pack on thermal responses during bicycle exercise. Environ Ergon. 2005:3:113-9. 
34. Bouskill L, Parsons K. Effectiveness of a neck cooling personal conditioning unit at reducing thermal strain during heat stress. In: Robertson SA ed. Proc Ann Conf Ergon Soc, Leicester, UK; 1996. pp. 236-42.

35. Gordon NF, Bogdanffy GM, Wilkinson J. Effect of a practical neck cooling device on core temperature during exercise. Med Sci Sports Exerc. 1990;22:245-9.

36. Kielblock AJ, van Rensburg JP, Franz RM. Body cooling as a method for reducing hyperthermia: an evaluation of techniques. S Afr Med J. 1986:69:378-80

37. Levels K, Teunissen LPJ, de Haan A, de Koning JJ, van Os B, Daanen HAM. Effect of warm-up and precooling on pacing during a 15-km cycling time trial in the heat. Int J Sport Physiol Perform. 2013;8:307-11.

38. Coelho LGM, Ferreira-Júnior JB, Williams TB, Maia-Lima A, Borba DA, Silva $\mathrm{CD}$, et al. Head pre-cooling improves 5-km time-trial performance in male amateur runners in the heat. Scand J Med Sci Sports. 2021;31(9):1753-63.

39. Walters P, Thom N, Libby K, Edgren S, Azadian A, Tannous D, Sorenson E, Hunt B. The effect of intermittent head cooling on aerobic performance in the heat. J Sport Sci Med. 2017;16:77-83.

40. Minett GM, Duffield R, Marino FE, Portus M. Volume-dependent response of precooling for intermittent-sprint exercise in the heat. Med Sci Sport Exerc. 2011;43:1760-9.

41. Hyde JL. The influence of head cooling combined with various cooling modalities on cooling rate after exercise in the heat. Master Thesis, University of Connecticut; 2015.

42. Desruelle AV, Candas V. Thermoregulatory effects of three different types of head cooling in humans during a mild hyperthermia. Eur J Appl Physiol. 2000;81:33-9.

43. Watanuki S. Effects of head cooling on cardiovascular and body temperature responses during submaximal exercise. Ann Physiol Anthrop. 1993;12:327-33.

44. Katsuura T, Ise N, Onoda H, Okada A, Kikuchi Y. Effect of head cooling on man at rest and during exercise under heat stress. Ann Physiol Anthrop. 1989:8:49-50

45. Greenleaf JE, van Beaumont W, Brock PJ, Montgomery LD, Morse JT, Shvartz E, Kravik S. Fluid-electrolyte shifts and thermoregulation: rest and work in heat with head cooling. Aviat Space Environ Med. 1980;51:747-53.

46. Stevens CJ, Kittel A, Sculley DV, Callister R, Taylor L, Dascombe BJ. Running performance in the heat is improved by similar magnitude with pre-exercise cold-water immersion and mid-exercise facial water spray. J Sport Sci. 2017;35:798-805.

47. Schlader ZJ, O'Leary MC, Sackett JR, Johnson BD. Face cooling reveals a relative inability to increase cardiac parasympathetic activation during passive heat stress. Exp Physiol. 2018;103:701-13.

48. Schlader ZJ, Coleman GL, Sackett JR, Sarker S, Johnson BD. Sustained increases in blood pressure elicited by prolonged face cooling in humans. Am J Physiol Regul Integr Comp Physiol. 2016;111:R643-8.

49. Mündel T, Bunn SJ, Hooper PL, Jones DA. The effects of face cooling during hyperthermic exercise in man: evidence for an integrated thermal, neuroendocrine and behavioural response. Exp Physiol. 2007;92(1):187-95.

50. Mündel T, Hooper PL, Bunn SJ, Jones DA. The effects of face cooling on the prolactin response and subjective comfort during moderate passive heating in humans. Exp Physiol. 2006;91:1007-14.

51. Armanda-da-Silva PAS, Woods J, Jones DA. The effect of passive heating and face cooling on perceived exertion during exercise in the heat. Eur J Appl Physiol. 2004;91:563-71.

52. Williams PA, Kilgour RD. Cardiovascular responses to facial cooling during low and moderate intensity exercise. Eur J Appl Physiol Occup Physiol. 1993:67:53-8.

53. Kratzing CC, Cross RB. Effects of facial cooling during exercise at high temperature. Eur J Appl Physiol Occup Physiol. 1984;53:118-20.

54. Riera F, Monjo R, Coudevylle GR, Meric H, Hue O. Face cooling during swimming training in tropical condition. Front Psychol. 2021;12:622184.

55. Miyazawa T, Mizutani M, Sheahan JP, Ichikawa D. Intermittent face cooling reduces perceived exertion during exercise in a hot environment. J Physio Anthropol. 2021:40:12.

56. Simmons SE, Mündel T, Jones DA. The effects of passive heating and head-cooling on perception of exercise in the heat. Eur J Appl Physiol. 2008;104:281-8.

57. Vargas NT, Chapman CL, Johnson BD, Gathercole R, Schlader ZJ. Skin wettedness is an important contributor to thermal behavior during exercise and recovery. Am J Physiol Regul Integr Comp Physiol. 2018;315(5):R925-33.
58. Lei TH, Wang F. Looking ahead of 2021 Tokyo Summer Olympic Games: how does humid heat affect endurance performance? Insight into physiological mechanism and heat-related illness prevention strategies. JTherm Biol. 2021;99:102975

59. Wiewelhove T, Conradt F, Rawlins S, et al. Effects of in-play cooling using simulated tennis match play in the heat on performance, physiological and perceptual measures. J Sports Med Phys Fitness. 2021;61(3):372-9.

60. Palmer CD, Sleivert GG, Cotter JD. The effects of head and neck cooling on thermoregulation, pace selection, and performance. Proc Aust Physiol Pharmacol Soc. 2001;32(Suppl 1):122.

61. Gordon RJFH, Tillin NA, Tyler CJ. The effect of head and neck per-cooling on neuromuscular fatigue following exercise in the heat. Appl Physiol Nutr Metab. 2020;45(11):1238-46.

62. Goosey-Tolfrey V, Diaper NJ, Crosland J, Tolfrey K. Fluid intake during wheelchair exercise in the heat: effects of localized cooling garments. Int J Sport Physiol Perform. 2008;3:145-56.

63. Simmons SE, Saxby BK, McGlone FP, Jones DA. The effect of passive heating and head cooling on perception, cardiovascular function and cognitive performance in the heat. Eur J Appl Physiol. 2008;104:271-80.

64. Ronald B, Shapiro R, Murphy M, Levenhagen D. Effectiveness of a commercial head-neck cooling device. J Strength Cond Res. 1999;13:198-205.

65. Douzi W, Dugué B, Vinches L, Al Sayed C, Hallé S, Bosquet L, Dupuy O. Cooling during exercise enhances performance, but the cooled body areas matter: a systematic review with meta-analyses. Scand J Med Sci Sports. 2019;29:1660-76.

66. Ruddock A, Robbins B, Tew G, Bourke L, Purvis A. Practical cooling strategies during continuous exercise in hot environments: a systematic review and meta-analysis. Sports Med. 2017:47:517-32.

67. Selkirk GA, McLellan TM. Influence of aerobic fitness and body fatness on tolerance to uncompensable heat stress. J Appl Physiol. 2001;91(5):2055-63.

68. Tanaka T. Gels. Sci Am. 1981;244(1):124-38.

69. Vargas NT, Chapman CL, Sackett JR, Abdul-Rashed J, McBryde M, Johnson $\mathrm{BD}$, et al. Thermal behavior remains engaged following exercise despite autonomic thermoeffector withdrawal. Physiol Behav. 2018;188:94-102.

70. Schlader ZJ, Stannard SR, Mündel T. Evidence for thermoregulatory behavior during self-paced exercise in the heat. J Therm Biol. 2011;36(7):390-6.

71. Tikuisis P, McLellan TM, Selkirk G. Perceptual versus physiological heat strain during exercise-heat stress. Med Sci Sports Exerc. 2002;34(9):1454-61.

72. Hasegawa $\mathrm{H}$, Cheung SS. Hyperthermia effects on brain function and exercise capacity. J Phys Fitness Sports Med. 2013;2(4):429-38.

73. Lei TH, Stannard SR, Perry BG, Schlader ZJ, Cotter JD, Mündel T. Influence of menstrual phase and arid vs. humid heat stress on autonomic and behavioral thermoregulation during exercise in trained but unacclimated women. J Physiol. 2016;595(9):2823-37.

74. Stevens JC, Choo KK. Temperature sensitivity of the body surface over the life span. Somatosens Motor Res. 1998:15(1):13-28.

75. Filingeri $\mathrm{D}$, Zhang $\mathrm{H}$, Arens EA. Thermosensitivity micromapping of warm and cold sensitivity across glabrous and hairy skin of male and female hands and feet. J Appl Physiol. 2018;125(3):723-36.

76. Grahn DA, Cao VH, Heller HC. Heat extraction through the palm of one hand improves aerobic exercise endurance in a hot environment. J Appl Physiol. 2005;99(3):972-8

77. Grahan DA, Cao VH, Nguyen CM, Liu MT, Heller HC. Work volume and strength training responses to resistive exercise improve with period heat extraction from the palm. J Strength Cond Res. 2012;26(9):2558-69.

78. Amorim FT, Yamada PM, Robergs RA, Schneider SM. Palm cooling does not reduce heat strain during exercise in a hot, dry environment. Appl Physiol Nutr Metab. 2010;35(4):480-9.

79. Scheadler CM, Saunders NW, Hanson NJ, Devor ST. Palm cooling does not improve running performance. Int J Sports Med. 2013:34(8):732-5.

\section{Publisher's Note}

Springer Nature remains neutral with regard to jurisdictional claims in published maps and institutional affiliations. 